\title{
Transient and thermal contact analysis for the elastic behavior of functionally graded brake disks due to mechanical and thermal loads
}

\begin{abstract}
In this paper, the transient and contact analysis of functionally graded (FG) brake disk is presented. The analysis was carried out using ANSYS parametric design language (APDL). The FG brake disk is made of metal-ceramic material. The material properties vary in radial direction with the values from full-metal at the inner radius to that of full-ceramic at the outer radius. In the analysis, FG brake disk is in contact with one pure pad disk and coulomb contact friction is considered as heat source. The non-dimensional results are obtained for specific value of grading index $(\mathrm{n}=1)$ by considering different material property divisions of 25, 50, 100 and 200. The results presented are for the pressure distribution, total stress, pad penetration, friction stress, heat flux and temperature during contact, for different values of contact stiffness factor, Fkn, which depends on the property gradation of FG brake disk with 200 material property divisions. The results show that the contact pressure and contact total stress increase with increasing values of Fkn, and hence it can be concluded that gradation of the metal-ceramic has significant effect in the thermomechanical response of FG brake disks.
\end{abstract}

